Short Paper

\title{
Mathematical Approach to Virtual Population Analysis*
}

\section{Tatsuro Akamine}

National Research Institute of Fisheries Science, Fukuura, Kanazawa, Yokohama 236, Japan

\author{
(Received May 16, 1994)
}

Key words: cohort analysis, VPA, mortality rate

Virtual Population Analysis (VPA) is a method of obtaining fishing mortality coefficients and stock sizes by solving the fishing equations. ${ }^{1)}$ The solution of VPA must be obtained by iteration because VPA is a non-linear model. In this paper, we consider a new method using mortality rates instead of coefficients, and obtain a simple algorithm to solve the equations and to evaluate the error of the solution easily.

VPA requires the solution of the following simultaneous equations:

$$
\begin{gathered}
C_{i}=N_{i} \frac{F_{i}}{F_{i}+M}\left\{1-\exp \left(-F_{i}-M\right)\right\} \\
N_{i+1}=N_{i} \exp \left(-F_{i}-M\right)
\end{gathered}
$$

where $C$ and $N$ are catch and stock sizes, $F$ and $M$ are fishing and natural mortality coefficients, and $i$ is age. Forward calculation obtains $F_{2} \sim F_{n}$ and $N_{1} \sim N_{n}$ using $C_{1} \sim C_{n}, M$ and $F_{1}$. In the backward calculation, we use $F_{n}$ (terminal $F$ ) instead of $F_{1}$, and obtain $F_{1} \sim F_{n-1}$ in the same way.

Equation (1) gives the following definition of fishing and natural mortality rates:

$$
\begin{aligned}
& E=\frac{F}{F+M}\{1-\exp (-F-M)\} \\
& D=\frac{M}{F+M}\{1-\exp (-F-M)\}
\end{aligned}
$$

These equations give the survival rate:

$$
S=\exp (-F-M)=1-E-D
$$

and the following inverse transformations: ${ }^{2)}$

$$
\begin{aligned}
& F=-\frac{E}{E+D} \ln (1-E-D) \\
& M=-\frac{D}{E+D} \ln (1-E-D)
\end{aligned}
$$

We can rewrite Eqs. (1) and (2) using Eqs. (3) and (4) as follows:

$$
\begin{gathered}
C_{1}=N_{1} E_{1}, \quad C_{2}=N_{2} E_{2} \\
N_{2}=N_{1}\left(1-E_{1}-D_{1}\right)
\end{gathered}
$$

These equations give the following solutions:

$$
E_{2}=\frac{C_{2} E_{1}}{C_{1}\left(1-E_{1}-D_{1}\right)}
$$

$$
E_{1}=\frac{C_{1} E_{2}\left(1-D_{1}\right)}{C_{1} E_{2}+C_{2}}
$$

Equation (10) gives the forward calculation, and Eq. (11) gives the backward calculation. The general case is given by changing the subscripts from $1 \rightarrow i$, and $2 \rightarrow(i+1)$.

From these equations, we obtain a simple method of solving Eqs. (1) and (2) provided that $D_{i}$ is known. However, this new algorithm gives a different solution for VPA if $M$ is constant. It is not natural to use $F$ and $M$, which are defined for the continuous model originally, for the discrete model (1) and (2). The condition of constant $M$ is less realistic than the condition of "known $M$ ". Therefore, this new method is more natural and realistic than VPA.

Next, we consider the error evaluation. The differential of Eq. (8) gives

$$
\frac{\mathrm{d} C}{C}=\frac{\mathrm{d} N}{N}+\frac{\mathrm{d} E}{E}=0
$$

This gives the following approximation:

$$
\frac{\Delta N}{N}=-\frac{\Delta E}{E}
$$

On the other hand, the differential of Eq. (9) gives

$$
\frac{\mathrm{d} N_{2}}{N_{2}}=\frac{\mathrm{d} N_{1}}{N_{1}}-\frac{\mathrm{d} E_{1}}{1-E_{1}-D_{1}}
$$

Because Eq. (12) shows that $\mathrm{d} N$ has the opposite sign to $\mathrm{d} E$, Eq. (14) gives the following approximation:

$$
\left|\frac{\Delta N_{2}}{N_{2}}\right|>\left|\frac{\Delta N_{1}}{N_{1}}\right|
$$

Backward calculation is considered to be better than forward calculation, because Eqs. (13) and (15) show that the relative error of $N_{i}$ increases in the forward calculation. This is a general theory of numerical analysis. However, Eqs. (1) and (2) are only equations and not a stochastic model. Therefore, Eqs. (13) and (15) do not suggest the mathematical superiority of the backward calculation.

The author wishes to thank Mr. K. Ishioka of Nansei National Fisheries Research Institute, and Dr. K. Hiramatsu of National Research Institute of Far Seas Fisheries for important information.

\section{References}

1) B. A. Megrey: American Fisheries Society Symposium, 6, 8-48 (1989).

2) T. Akamine: Nihonkai-ku Suisan Siken Kenkyuu Renraku News, 354, 7-9 (1990) (in Japanese).

\footnotetext{
* Contribution B No. 101 from the National Research Institute of Fisheries Science.
} 